\title{
Use of a telenursing triage service by Victorian parents attending the emergency department for their child's lower urgency condition
}

Doctor Erin Turbitt, $\mathrm{PhD}$,

Professor Gary Lee Freed, MD MPH

Author contributions: E Turbitt made substantial contributions to analysis and interpretation of the data; drafting and revising the work. GL Freed made substantial contributions to the conception and design of the work; interpretation of the data; drafting the work and revising. Both authors approve of the final version and are accountable for the integrity of the work.

Affiliations: Centre for Health Policy, Melbourne School of Population and Global Health, The University of Melbourne, Victoria, Australia

Correspondence:

Professor Gary L Freed, Director, Health Systems and Workforce Unit, Center for Health Policy, School of Population and Global Health, The University of Melbourne, Level 4, 207 Bouverie Street, Parkville 3010 VIC Australia $\mathrm{T}:+61390356335$

E:gary.freed@unimelb.edu.au

Word count: 2252 


\begin{abstract}
Objective: The Victorian Nurse-On-Call service has been in use for over eight years, though little research has been conducted investigating the service. This study aimed to explore whether parents in Victoria presenting with their child to the emergency department (ED) for lower urgency conditions use the NOC prior to ED arrival and whether the advice given impacts their decision to attend the ED.
\end{abstract}

Methods: A survey study of 1150 parents attending one of four EDs in Victoria, Australia for their child's lower urgency condition.

Results: Few parents (20\%) contacted the service before attending. Of those who did contact the service, $70 \%$ were instructed to attend the ED. Parents reported they did not contact the service due to lack of awareness (16\%) and because they perceive the service to not be helpful (53\%).

Conclusions: The findings of our study show that use and awareness of NOC is low in parents attending the ED for their child's lower urgency condition. The success of NOC in the goal of deferring non-urgent conditions from presenting to the ED appears limited. Telenursing triage services in Australia should consider assessment of their algorithms to increase the likelihood that where appropriate, lower urgency conditions are directed to primary care services rather than the ED.

Keywords: emergency medicine, health services, low urgency, nurses, paediatric, parent, telephone triage

\title{
INTRODUCTION
}

Patient telephone triage is one strategy to manage demand for healthcare services, including emergency department (ED) presentations. ${ }^{1}$ Telephone triage is generally conducted 
by trained nurses who give health advice to callers, usually leading to one of three outcomes; self-care, general practitioner (GP) visit, or ED presentation. ${ }^{2}$

A standardised telenursing triage service (HealthDirect) was first introduced to Australia in May 1999 in Western Australia. ${ }^{3}$ The national HealthDirect service is now operational in all Australian states, except Victoria and Queensland where each state operates their own service. The Victorian Nurse-On-Call (NOC) service, initiated in June 2006, operates 24 hours a day, 7 days a week. In 2010, an audit reported that the service receives about 1000 calls per day. ${ }^{2}$ Nurses must have a minimum of three years clinical nursing experience. As with many telenursing triage services, NOC nurses use structured algorithms to assist with disposition of the calls. The NOC service is operated by Medibank Health Solutions under contract to the State of Victoria.

There is evidence of increasing ED presentations for children in Victoria and Australia. Data from the Victorian Emergency Minimum Dataset (VEMD) show an increase of 29\% in ED presentations from 2002 to 2013 for the $0-4$ age group. ${ }^{4}$ Of note, $24 \%$ of callers to the NOC service are parents/carers wanting advice about children aged $1-4 .^{2} \mathrm{~A}$ frequently stated benefit of a State providing teletriage services is a reduction in ED presentations, though evidence in this area is mixed. Most studies have shown that phone consultations have no impact, or may increase ED presentations, ${ }^{5,6}$ though one Australian study suggested $78 \%$ of patients planning to attend the ED decided not to as a result of contacting a telephone triage service. ${ }^{7}$

Most previous research of telenursing triage systems in Australia has focused on caller satisfaction, caller adherence to advice, and appropriateness of advice offered. Further, studies generally do not present data stratified by age group. Patients generally report being very satisfied with the service. ${ }^{8}$ While one study reported high levels ( $79 \%$ of callers) of compliance with the advice given, ${ }^{9}$ a more recent 2012 study showed that $52 \%$ of callers attended the ED although they were given advice to see a GP. ${ }^{10}$ A 2010 study carried out by the Department of 
Health, Victoria showed that $68 \%$ of callers to the Victorian NOC comply with advice. ${ }^{2}$ Studies investigating the appropriateness or quality of advice given through Australian telenursing services have produced varied results. One study reported that correct advice is given in $69 \%$ of cases, ${ }^{11}$ with another finding that $79 \%$ of recommendations to HealthDirect callers were assessed as appropriate. ${ }^{10}$ Mystery call testing to the NOC service in 2009-2010 showed that $2 \%$ of callers were given 'unsafe' advice, most commonly due to nurses failure to pick up on callers' cues. ${ }^{2}$

While it is important for a service such as NOC to have high levels of satisfaction among patients and to give safe advice, from a resource perspective it is important that the service reduces the demand for ED services when appropriate. There is a lack of paediatric Australian studies on this topic. The study presented here aimed to investigate whether parents in Victoria presenting with their child to the ED for lower urgency conditions use the NOC prior to ED arrival and whether the advice given impacts their decision to attend the ED. We also investigated potential reasons why some parents presenting to the ED do not use the service.

\section{METHODS}

This study is part of a larger project to investigate reasons for increasing numbers of children presenting to EDs in Victoria, Australia for lower urgency conditions.

\section{Sample and recruitment}

The sample consisted of parents or guardians of patients ( $<9$ years of age) attending the ED at one of four Victorian hospitals. Recruitment was carried out at three public general hospitals and one specialist children's hospital in metropolitan Melbourne from May November 2014. Only parents whose child was assigned an Australasian Triage System triage 
category of 4 or 5 (lower urgency) were invited to take part. Parents who were in obvious distress were not approached. Those with limited English proficiency were ineligible to participate. Potential participants were approached by trained research assistants and provided with an information letter before being invited to answer the survey. On completion of the survey, participants were offered a $\$ 10$ gift card to a local supermarket chain in appreciation for their time.

\section{Survey}

The survey instrument was developed based on a review of literature and expert consultation with ED directors at all four hospitals. Following initial development, the survey was pilot tested with 10 parents at the study hospitals. The survey contained 81 items and used branching logic to tailor specific questions to follow selected answers. The questionnaire used single and multiple response options as well as Likert-scale responses to specific questions.

\section{Data collection}

Parents who agreed to participate signed a consent form and completed the survey electronically using an iPAD supplied to them. Study data were collected and managed using REDCap electronic data capture tools hosted at The University of Melbourne. ${ }^{12}$ Surveys were administered in approximately equal numbers across three time periods (8am-4:59pm; 5pm9:59pm; 10pm-7:59am) and on weekends at all hospitals.

\section{Data analysis}

The four survey questions directly related to the NOC service were analysed for this report (Supplementary Figure 1). Statistical analyses were performed using STATA 13.0 (StataCorp College Station, TX). Frequency and descriptive analyses were initially undertaken to examine the data. Subsequently, bivariate analyses using chi-square statistics were conducted to determine the differences, if any, among respondents based on birth order of the child they 
brought to the ED (first born vs. all other), age of the primary carer ( $\leq 30$ vs. $>30$ years), household income $(\leq 100 \mathrm{~K}$ vs. $>100 \mathrm{~K})$ and the time band of presentation to the ED.

This project received ethics approval from The University of Melbourne and all hospital Human Research Ethics Committees. Responses were confidential, voluntary and anonymous.

\section{RESULTS}

There were 1150 parents who participated in the study with a decline rate of $19.9 \%$. This included 826 responses from parents who attended the three public general hospitals and 324 who attended the specialist children's hospital. Table 1 shows a summary of the characteristics of participating parents, including time of presentation to the ED. A small number of parents did not respond to all items.

Of the 1150 participants, only 230 (20\%) indicated they tried to call NOC before bringing their child with a lower urgency condition to the ED. Younger parents (under 30) were more likely to call the service than parents over 30 ( $24 \%$ vs. $18 \%$; $P=0.04)$. More parents attending the ED at night had tried to call the NOC service compared to those presenting at other time bands ( $31 \%$ vs. $17 \%$ during the day, $19 \%$ in the evening, and $18 \%$ on the weekend; $P=0.001$ ). A higher number of parents made a call to NOC if their child's chief complaint was illness, compared to parents whose children had injuries ( $25 \%$ vs. $10 \% ; P<0.001)$. Most parents who did call the NOC service found that call helpful (85\%).

Parents who called NOC before coming to the ED were asked to give reasons why they attended the ED following that call. Table 2 shows their responses (respondents could select more than one answer). Most callers (70\%) came to the ED because they were told to do so by NOC. A further $22 \%$ came because they were still worried about their child after the call. There were no statistically significant differences among parents based on their demographic characteristics or time of ED visit. 
For those parents who did not call NOC prior to presenting to the ED, we queried as to why they did not do so (Table 3). Responses were varied, with the top three reasons being: parents did not think it would be helpful (43\%, with a further $10 \%$ having tried NOC in the past and found it not helpful); they are always told to attend the ED by NOC anyway (20\%); and they had not heard of the service (16\%). Parents aged over 30 were more likely to think the call would not be helpful than parents 30 and under (47\% vs. $32 \%$; $P<0.001)$. Compared to parents whose child had an illness, more parents whose child presented to the ED with an injury did not call because they thought the call would not be helpful (55\% vs. $35 \% P<0.001)$.

\section{DISCUSSION}

The most important finding of our study is that only a small proportion of parents attending the ED for their child's lower urgency condition contacted NOC. Additionally, of those who did call prior to attending, most presented to the ED as advised to do so by the service. Parents who did not contact NOC mostly thought it would not be helpful, that they would be sent to the ED anyway, or had not heard of the service.

The NOC service was established in 2006 with the primary aim to provide the community with rapid access to reliable health information. A secondary aim was to relieve the demand for ED services. ${ }^{2}$ From our results, we are not able to determine whether NOC has impacted the demand for ED services. However, our finding that $70 \%$ of those who called prior to attending the ED for their child's lower urgency condition were advised to attend, suggests there may be patients referred to ED by NOC who could potentially be managed outside of an emergency setting. This is an issue of particular importance given that presentations to ED by children in Victoria are increasing. ${ }^{4}$

A major challenge for a service such as NOC is that it must be sensitive enough to correctly refer emergencies to the ED and avoid adverse events, though specific enough to identify cases that do not require urgent care. ${ }^{13}$ A number of studies have investigated the quality of advice provided by triage call centre services in Australia. These investigations 
generally report a high level of safety, defined as low rates of adverse events following patient contact with the service. ${ }^{2,14,15}$ Although a high level of safety could be the result of the services only receiving calls for lower severity conditions, a 2010 study suggests this is not the case for the USA's Ask Mayo Clinic telephone triage service. ${ }^{16}$

Another explanation for high levels of safety may be that automated algorithm systems used by teletriage systems have minimal ability to detect which cases require less urgent evaluation. Studies from the USA have similarly identified significant numbers of callers who are inappropriately directed to attend EDs when care provided by outpatient doctors or self-care would suffice. ${ }^{13,17}$ It may be appropriate for telenursing triage services in Australia to reevaluate their algorithms to increase the likelihood that where appropriate, non-urgent conditions are directed to primary care services.

Similar to our study, an investigation from 2008 focusing on parents' health information seeking behaviours showed that few (10\%) used NOC prior to attending the ED. ${ }^{18}$ This is most likely a consequence of parents' lack of awareness of the service, and their prior perspectives about the service not being helpful. A study from the UK reported that $40 \%$ of outpatients surveyed were unaware of 'NHS Direct', the UK telenursing triage service. ${ }^{19} \mathrm{We}$ found that $16 \%$ of respondents had not heard of NOC.

Our results are similar to others showing that telenursing services are used more during after-hours ${ }^{20}$ and by younger individuals ${ }^{21}$. We also found that parents are more likely to contact NOC before attending the ED for child illnesses rather than injuries. However, the overall pattern across all time bands and ages is that most parents presenting to the ED with children having lower urgency conditions do not contact NOC.

To increase the number of people using NOC prior to ED presentation, state and federal officials should consider a broader publicity campaign. These campaigns could aim to capture the attention of parents (of all ages) and ensure the purpose of NOC is clearly articulated, including that it is also appropriate for injury related events. Advertising for NOC to raise awareness could be undertaken at EDs as well as GP surgeries, as our results show that a 
significant number of parents attending the ED for their child's lower urgency condition have not heard of the service.

A more vexing issue is the perceptions of $53 \%$ of respondents who are aware of the service but did not think it would be helpful. The reputation of a healthcare service may be a result of prior personal experience with that service or from other second-hand sources. Efforts beyond simple awareness campaigns will be required to address this issue.

\section{Limitations:}

Our study only captured the perspectives of those parents who actually attended the ED, therefore these results may not be generalisable to all callers to the service. Additionally, this study was limited to parents of children who had been triaged as lower urgency and the experiences of relatively high urgency conditions may differ. This study relied on respondents to recall information and there is potential for some respondents to do so incorrectly. Future studies could record advice provided by NOC and track subsequent parental behaviour. Finally, the participants in our study were limited to those with English proficiency. These limitations aside, this study is one of the few independent studies of an Australian nurse teletriage service. Many previous studies have been commissioned by or affiliated in some way to service providers. . $^{3,14,15,20}$

\section{Conclusions:}

The findings of our study demonstrate that the success of the NOC service in the goal of deferring non-urgent conditions from presenting to the ED appears limited. In addition, a significant portion of parents presenting to the ED with a child having lower urgency conditions have not heard of the service and an even larger proportion do not believe the service would have been helpful.

\section{ACKNOWLEDGEMENTS}

We thank Gemma Catley for her administrative assistance in the submission of this manuscript. 
This study was funded by the Victorian Government's Department of Health, Royal Children's Hospital Foundation, Inner Northwest Medicare Local and the Northern Melbourne Medicare Local. 


\section{REFERENCES}

1. Department of Health and Aging. COAG Health Services: National Health Call Centre Network Health budget 2006-2007. 2007 [cited March 2015]. Available from: https://www.healthyactive.gov.au/internet/budget/publishing.nsf/Content/budget2006hfact59.htm.

2. Victorian Auditor-General. Delivery of Nurse-on-call. Victoria: Victorian AuditorGeneral's Office: 2010.

3. St George I, Cullen M, Gardiner L, et al. Universal telenursing triage in Australia and New Zealand: A new primary health service. Australian family physician. 2008;37:476 - 479.

4. Freed GL, Gafforini S, Carson N. Age distribution of emergency department presentations in Victoria. Emerg Med Australas. 2015:1-6.

5. Bunn F, Byrne G, Kendall S. The effects of telephone consultation and triage on healthcare use and patient satisfaction: a systematic review. Br J Gen Pract. 2005;55:956-961.

6. Nagree Y, Cameron P, Gosbell A, et al. Telephone triage is not the answer to ED overcrowding. Emerg Med Australas. 2012;24:123-126.

7. Bolton P, Gannon S, Aro D. HealthConnect: a trial of an after-hours telephone triage service. Aust Health Rev. 2002;25:95-103.

8. Keatinge D, Rawlings K. Outcomes of a nurse-led telephone triage service in Australia. Int J Nurs Pract. 2005;11:5-12.

9. Sprivulis P, Carey M, Rouse I. Compliance with advice and appropriateness of emergency presentation following contact with the HealthDirect telephone triage service. Emerg Med Australas. 2004;16:35-40.

10. Ng JY, Fatovich DM, Turner VF, et al. Appropriateness of healthdirect referrals to the emergency department compared with self-referrals and GP referrals. Med J Aust. 2012;197:498-502.

11. Andrews J, Kenneth L, Jennifer A. Professional telephone advice to parents with sick children: Time for quality control!. J Paediatr Child Health. 2002;38.

12. Harris PA, Taylor R, Thielke R, et al. Research electronic data capture (REDCap) - a metadata-driven methodology and workflow process for providing translational research informatics support. Journal of biomedical informatics. 2009;42:377-381.

13. Doctor K, Correa K, Olympia RP. Evaluation of An After-Hours Call Center: Are Pediatric Patients Appropriately Referred to the Emergency Department? Pediatr Emerg Care. 2014;30:798-804.

14. St George I, Baker J, Karabatsos G, et al. How safe is telenursing from home? Collegian. 2009;16:119-123.

15. St George I, Cullen M, Branney M. Healthline: do primary care doctors agree with the advice. N Z Med J. 2005;118:U1693.

16. North F, Varkey P. How serious are the symptoms of callers to a telephone triage call centre? J Telemed Telecare. 2010;16:383-388.

17. Scarfone RJ, Luberti AA, Mistry RD. Outcomes of children referred to an emergency department by an after-hours call center. Pediatr Emerg Care. 2004;20:367-372.

18. Khoo K, Bolt P, Babl FE, et al. Health information seeking by parents in the Internet age. J Paediatr Child Health. 2008;44:419-423.

19. Larner AJ. NHS Direct telephone helpline: frequency of use over time and by age and gender in an outpatient population. Telemedicine and e-health. 2009;15:199-201.

20. Turner VF, Bentley PJ, Hodgson SA, et al. Telephone triage in western Australia. Med J Aust. 2002;176:100-103.

21. Knowles E, O'Cathain A, Turner J, et al. Awareness and use of a new urgent care telephone service, NHS 111: cross-sectional population survey. J Health Serv Res Policy. 2014;19:224-230. 
Table 1: Respondent characteristics

\begin{tabular}{|c|c|c|}
\hline Characteristic & & $\%(\mathrm{~N})$ \\
\hline \multicolumn{3}{|c|}{ Time band of presentation $(\mathrm{N}=1150)$} \\
\hline & Day (8:00am-4:59pm) & $27(309)$ \\
\hline & Evening (5pm-9:59pm) & $27(308)$ \\
\hline & Night (10pm-7:59am) & $17(198)$ \\
\hline & Weekend & $29(335)$ \\
\hline \multicolumn{3}{|c|}{ Age of child $(\mathrm{N}=1143)$} \\
\hline & $<1$ years & $23(258)$ \\
\hline & $1-4$ years & $51(583)$ \\
\hline & $5-9$ years & $26(302)$ \\
\hline \multicolumn{3}{|c|}{ Household composition $(\mathrm{N}=1122)$} \\
\hline & Single parent & $14(162)$ \\
\hline & Dual parent & $82(917)$ \\
\hline & Other & $4(43)$ \\
\hline \multicolumn{3}{|c|}{ Household income $(\mathrm{N}=1095)$} \\
\hline & $0-\$ 25000$ & $13(137)$ \\
\hline & $\$ 25001-\$ 50000$ & $16(173)$ \\
\hline & $\$ 50001-\$ 80000$ & $23(251)$ \\
\hline & $\$ 80001-\$ 100000$ & $17(184)$ \\
\hline & $>\$ 100000$ & $32(350)$ \\
\hline \multicolumn{3}{|c|}{ Age of child's primary carer $(\mathrm{N}=1129)$} \\
\hline & $<20$ & $4(48)$ \\
\hline & $21-30$ & $27(306)$ \\
\hline & $31-40$ & $52(590)$ \\
\hline & $>40$ & $16(185)$ \\
\hline \multicolumn{3}{|c|}{ Chief complaint $(\mathrm{N}=1116)$} \\
\hline & Injury & $34(373)$ \\
\hline & Illness & $66(743)$ \\
\hline
\end{tabular}

Table 2: What were your reasons for coming to the ED after calling Nurse on Call? $(\mathrm{N}=\mathbf{2 3 0})$

\begin{tabular}{|lc|}
\hline & $\%(\mathbf{N})$ \\
\hline I was told by Nurse-On-Call service to go to the ED & $70(162)$ \\
\hline I was still worried about my child & $22(51)$ \\
\hline My child's condition worsened since the call & $11(25)$ \\
\hline My child's condition did not improve since the call & $8(18)$ \\
\hline I did not have confidence in the call centre to provide advice for this condition & $3(7)$ \\
\hline I was on hold for a long time and just hung up and came to the ED & $2(5)$ \\
\hline
\end{tabular}


Table 3: Why did you not try to call the Nurse-On-Call service and speak with a nurse? (N=916)

\begin{tabular}{|lc|}
\hline & $\%(\mathbf{N})$ \\
\hline I did not think it would be helpful & $43(393)$ \\
\hline They always tell me to go to the ED & $20(180)$ \\
\hline I have never heard of this service & $16(144)$ \\
\hline I have tried in the past and found it not helpful & $10(95)$ \\
\hline I have tried in the past and spent too long on hold & $6(57)$ \\
\hline I could not describe the problem over the phone & $6(54)$ \\
\hline I don't know how to call the Nurse-On-Call service & $4(39)$ \\
\hline I don't trust a nurse to give me advice & $2(15)$ \\
\hline I was worried I could not describe the problem in English & $1(13)$ \\
\hline
\end{tabular}

